\title{
Thermo Structural Analysis of Two Stroke Si Engine Cylinder
}

\author{
Dayadi Nageswararao ${ }^{1}$, Gobbur Mukesh ${ }^{2}$ \\ ${ }^{1,2}$ Department Of Mechanical Engineering ,Cmr Technical Campus ,Jntu ,India
}

\begin{abstract}
Cylinder is the heart of internal combustion engine as combustion takes place inside the cylinder large amount of heat is produce inside the cylinder due to that heat distortion of cylinder wall may takes place. Due to inadequate heat transfer through the engine cylinder block the engine cylinder gets overheated, lead to knocking and some time result into structural failure. This also causes an increase in the thermal stresses in the liner wall which ultimately affects the strength of liner wall. The main objective of this paper is to carry out thermo structural analysis of combustion chamber (Liner) in Ansys workbench 15.0 to predict temperature distribution across the combustion chamber of scavenged engine. The design of 2 stroke engine cylinder is modeled in ANSYS workbench 15.0. Around 27 cases are considered of different fin thicknesses, gaps and materials. Then geometry for each case is modeled. The boundary conditions are applied to each case. For thermal the boundary conditions are convection at liner, fins and exhaust section of the cylinder. For structural, cylindrical support and pressure are applied. Each case is analyzed in the ANSYS workbench 15.0. The results of total heat transfer and stresses induced in the cylinder for each case are obtained.
\end{abstract}

Keywords: Engine cylinder, Fin thicknesses, gaps and materials, Thermo structural analysis, ANSYS workbench 15.0.

\section{Introduction}

Heat transfer is a very wide field used in analysis of internal combustion engine heat transfer effect parameter such as performance, emission and also efficiency. It is said that for a given mass of fuel higher the heat transfer to the combustion wall will reduce the average combustion pressure and temperature, this indirectly reduces the work done by the piston per cycle and these effects the specific power. Temperature rise of the engine parts may cause a serious durability of the engine. The shape of isothermal lines and high temperature regions become more important in these studies. The experimental way will find these regions are costly and time consuming; Analytical methods are almost equally good for fast conformation of this region by using finite elements Measuring the actual dimension of various components of two-stroke S.I engine (BAJAJ, 100cc). modeling of piston, liner along with combustion chamber are done using Ansys 15 we analyzed the temperature distribution and thermal stresses on above component, compare that thermal stresses with theoretically calculated thermal stresses.

\section{Ii. Specification Of The Problem}

Objective of the paper is to design cylinder with fins for BAJAJ 100cc engine, by changing the fin thickness and distance between the fins to analyze the thermal properties of the fins. Analyzation is also done by varying the materials of fins. Present used material for cylinder fin body is cast iron .Our aim is to change the material for fin body by analyzing the fin body with other materials and also by changing the geometry distance between the fins and thickness of the fins. Thickness of fins are- 2, 2.5 and 3mm Distance between the fins -5.6 , $6.6 \mathrm{~mm}$. Materials are considered for analysis -Grey cast Iron, Mild steel ,Magnesium alloy and Aluminum alloy.

\subsection{Survey on air cooled engine cylinder fins, commercially:}

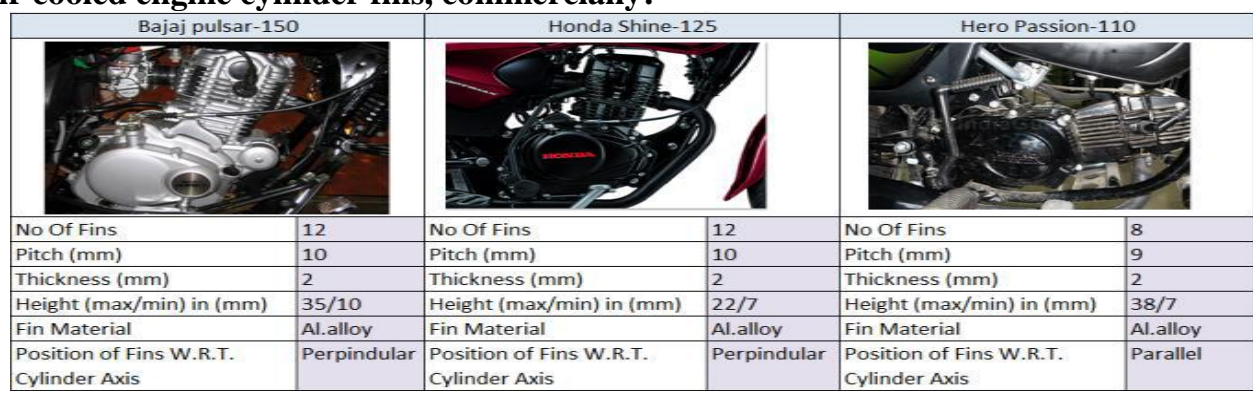

Fig 1: engine cylinder fins 


\section{III.Design}

3.1 Design parameters for selected model :Engine cylinder with fins is a complicated shape which was developed in professional software ANSYS Work bench 15.0.

Table 1: Properties of different materials

\begin{tabular}{|l|l|l|l|l|}
\hline Parameters & Mild steel & Grey cast iron & Magnesium alloy & Alluminium alloy \\
\hline $\mathrm{E}(\mathrm{Pa})$ & $2 \mathrm{E}+11$ & $1.1 \mathrm{E}+11$ & $4.5 \mathrm{E}+10$ & $7.1 \mathrm{E}+10$ \\
\hline Poisson ratio & 0.3 & 0.28 & 0.35 & 0.33 \\
\hline $\mathrm{K}(\mathrm{w} / \mathrm{m} 0 \mathrm{c})$ & 60.5 & 52 & 156 & 165 \\
\hline$\alpha(/ 0 \mathrm{c})$ & $1.2 \mathrm{E}-05$ & $1.1 \mathrm{E}-05$ & $2.6 \mathrm{E}-05$ & $2.3 \mathrm{E}-05$ \\
\hline $\mathrm{CP}(\mathrm{J} / \mathrm{Kg} 0 \mathrm{C})$ & 480 & 420 & 1013 & 875 \\
\hline$\rho(\mathrm{kg} / \mathrm{m} 3)$ & 7850 & 7272 & 1800 & 2770 \\
\hline
\end{tabular}

\subsection{Equations:}

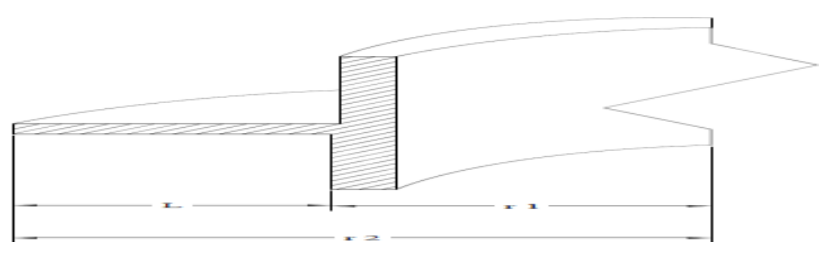

For a circumferential fin of rectangular cross section

$\mathrm{L}=\mathrm{r} 2-\mathrm{r} 1 \quad \mathrm{Lc}=\mathrm{L}+\mathrm{t} / 2 \quad \mathrm{r} 2 \mathrm{c}=\mathrm{r} 1+\mathrm{Lc} \quad \mathrm{Am}=(\mathrm{r} 2 \mathrm{c}-\mathrm{r} 1) \mathrm{t}$

Max Heat transferQ $=2 \pi \mathrm{h}\left(\mathrm{r}_{2}{ }^{2}-\mathrm{r}_{1}{ }^{2}\right) \theta_{0} \quad \theta_{0}=\left(\mathrm{t}_{\mathrm{h}}-\mathrm{t}_{\infty}\right)$

Heat transfer by the fin $\mathrm{Q}_{\mathrm{fin}}=\dot{\eta}_{\mathrm{fin}} \times \mathrm{Q}_{\max }$

Effectiveness $\in=\sqrt{ } p k / h A$

\section{IV.Analysis \& Optimization}

\subsection{Geometry of cylinder}

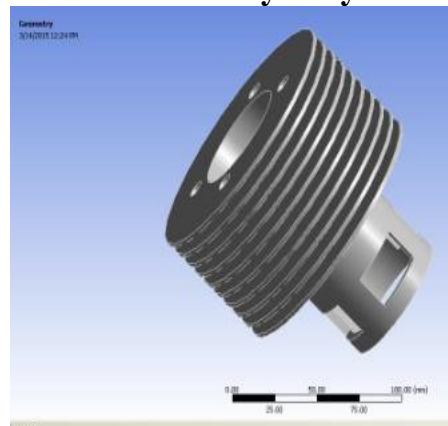

Fig 2: geometry of model
4.2 Meshing of cylinder geometry

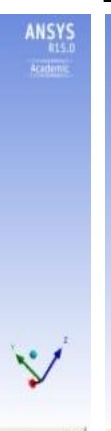

$\cdots$

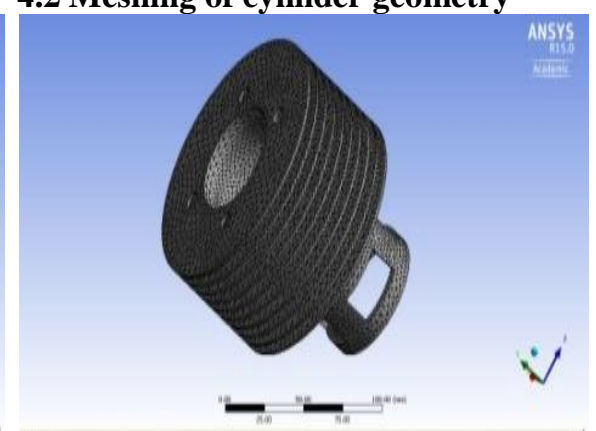

Fig 3: mesh model

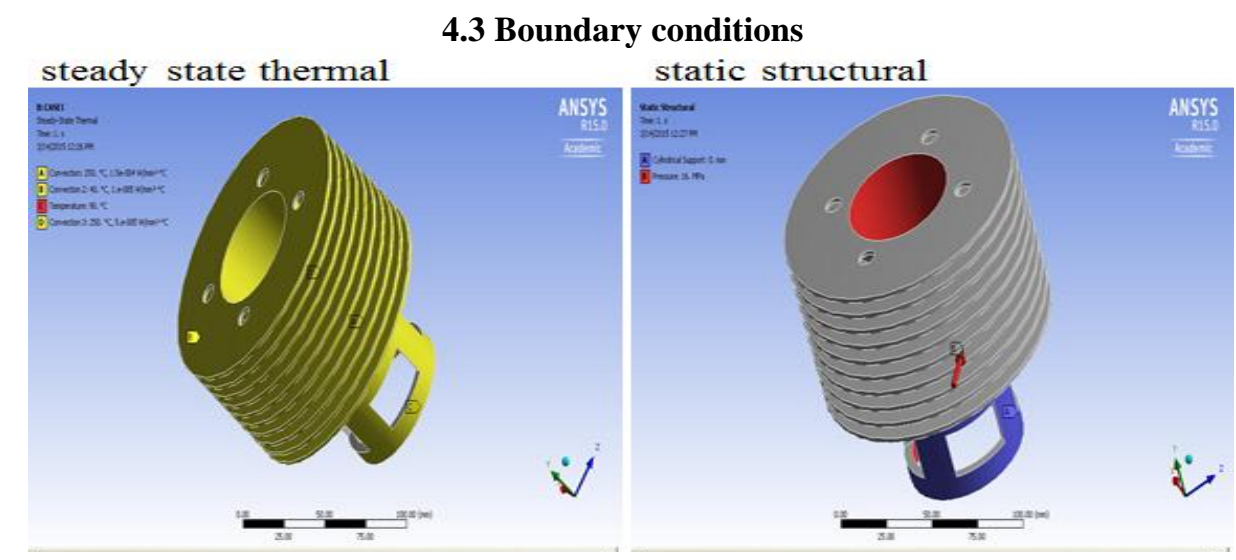

Fig 3: boundary condition 
4.4 Case 1:Material: Mild steel : Thermal analysis of cylinder

1. For the gap between the successive fins: $5.6 \mathrm{~mm}$

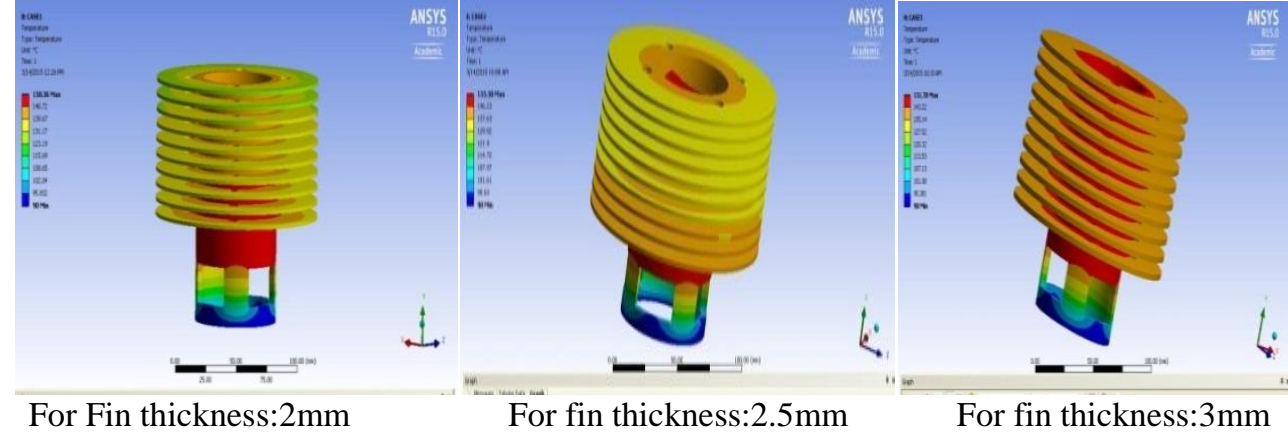

Fig 4

2. For the gap between the successive fins: 6.6

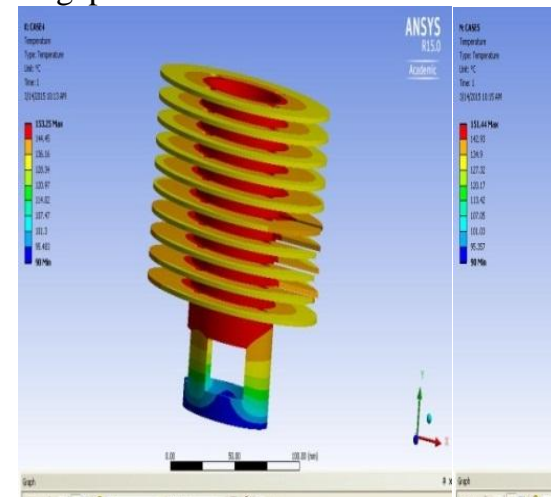

For Fin thickness:2mm

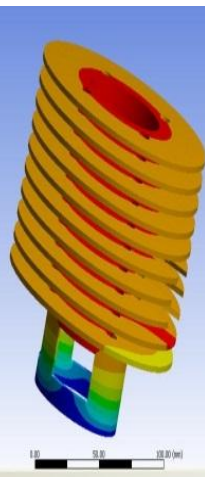

For fin thickness:2.5mm For fin thickness:3mm Fig 5

Table 2: Steady state thermal:

\begin{tabular}{|c|c|c|c|}
\hline Material & \multicolumn{1}{|c|}{$\begin{array}{l}\text { Gap between } \\
\text { fins(mm) }\end{array}$} & Fin thickness $(\mathbf{m m})$ & Temperature $\left({ }^{\circ} \mathbf{C}\right)$ \\
\hline \multirow{3}{*}{ Mild steel } & \multirow{2}{*}{5.6} & 2 & 158.36 \\
\cline { 2 - 3 } & & 2.5 & 155.38 \\
\cline { 2 - 3 } & \multirow{2}{*}{6.6} & 2 & 151.78 \\
\cline { 2 - 4 } & & 2.5 & 153.25 \\
\cline { 2 - 4 } & & 3 & 151.44 \\
\cline { 2 - 4 } & & & 151.42 \\
\hline
\end{tabular}

- Structural analysis:

3. For the gap between the successive fins: $5.6 \mathrm{~mm}$ :

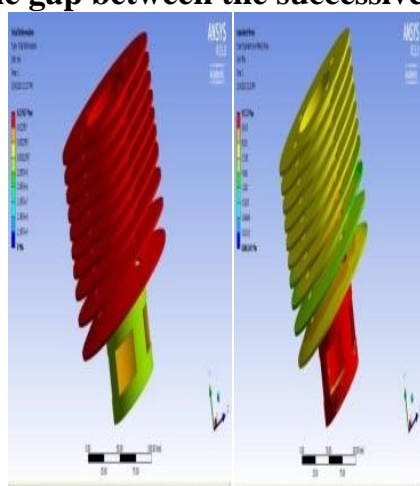

For fin thickness: $2 \mathrm{~mm}$

Fig 6

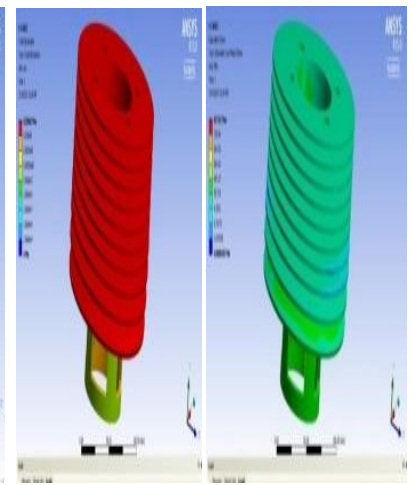

For fin thickness: $2.5 \mathrm{~mm}$ Fig 7

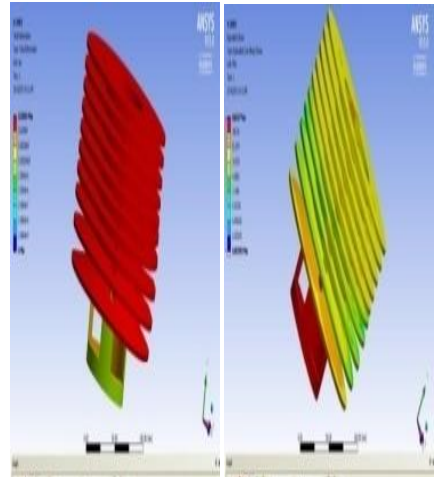

For fin thickness: $3 \mathrm{~mm}$ Fig 8 
4. For the gap between the successive fins: $6.6 \mathrm{~mm}$ :

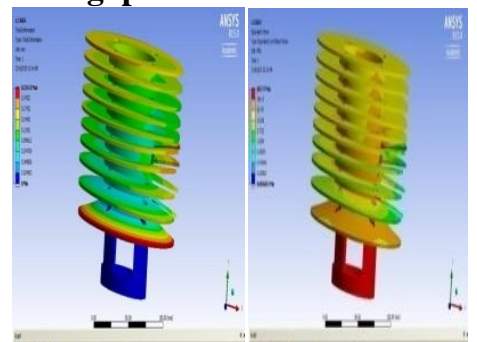

For fin thickness: $2 \mathrm{~mm}$

Fig 9

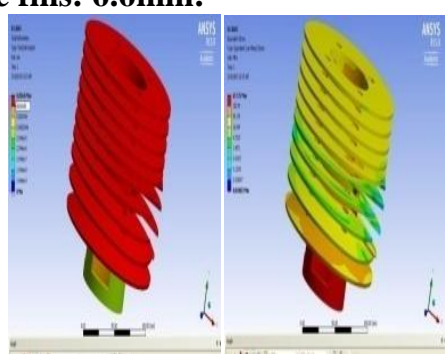

For fin thickness: $2.5 \mathrm{~mm}$ Fig 10

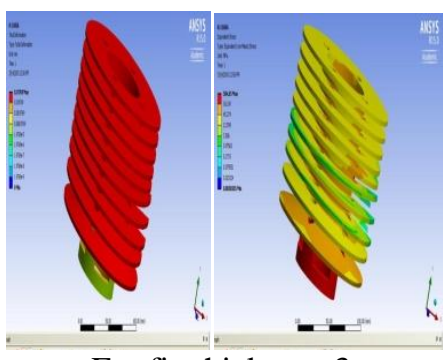

For fin thickness:3mm Fig 11

Table 3: static structural

\begin{tabular}{|c|c|c|c|c|}
\hline Material & Gapbetweenfins(mm) & $\begin{array}{l}\text { Finthickness(m } \\
\text { m) }\end{array}$ & $\begin{array}{l}\text { Totaldeformation(m } \\
\text { m) }\end{array}$ & $\begin{array}{l}\text { Equalentstress(M } \\
\text { pa) }\end{array}$ \\
\hline \multirow{3}{*}{ Mild steel } & \multirow{3}{*}{5.6} & 2 & 0.22957 & 917.57 \\
\cline { 3 - 5 } & & 2.5 & 0.28668 & 827.62 \\
\cline { 2 - 5 } & & 3 & 0.20869 & 660.87 \\
\cline { 2 - 5 } & \multirow{2}{*}{6.6} & 2 & 0.22413 & 682.71 \\
\cline { 2 - 5 } & & 2.5 & 0.20446 & 617.75 \\
\cline { 2 - 5 } & & 3 & 0.19769 & 584.65 \\
\hline
\end{tabular}

4.5 . Material: Grey cast iron: Thermal analysis of cylinder:

1. For the gap between the successive fins: $5.6 \mathrm{~mm}$

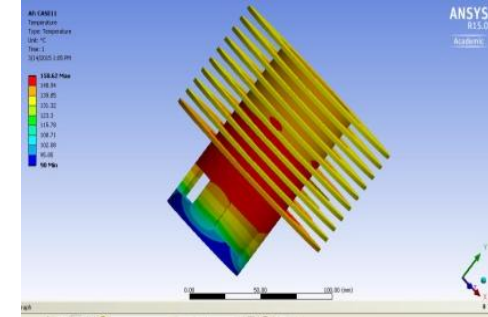

For Fin thickness:2mm

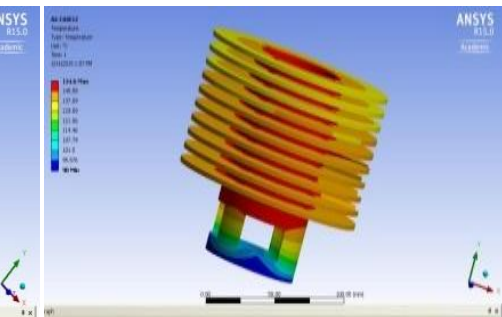

For fin thickness: $2.5 \mathrm{~mm}$

Fig 12

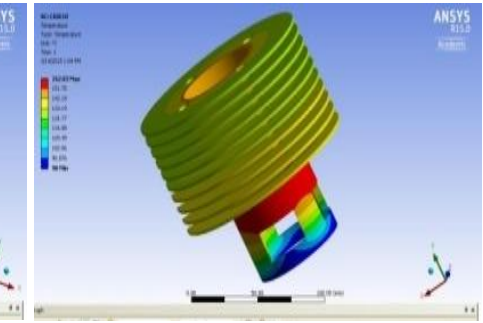

For fin thickness:3mm

2. For the gap between the successive fins: $6.6 \mathrm{~mm}$

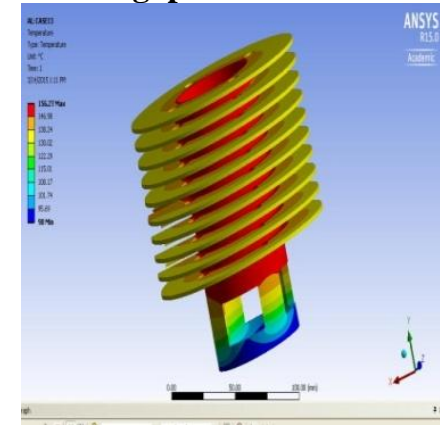

For Fin thickness:2mm

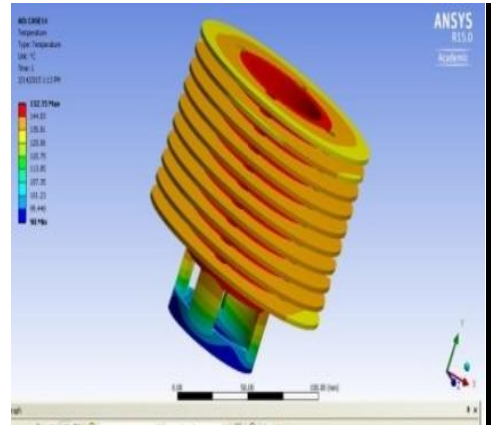

For fin thickness: $2.5 \mathrm{~mm}$

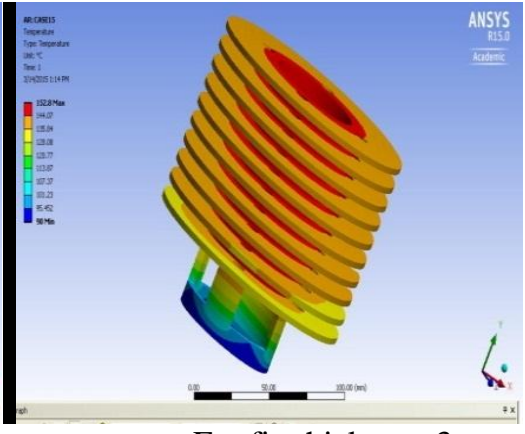

For fin thickness:3mm

Fig 13

Table 4: Steady state thermal:

\begin{tabular}{|c|l|l|l|}
\hline Material & $\begin{array}{l}\text { Gap between } \\
\text { fins(mm) }\end{array}$ & $\begin{array}{l}\text { Fin thickness } \\
(\mathbf{m m})\end{array}$ & Temperature $\left({ }^{\circ} \mathbf{C}\right)$ \\
\hline \multirow{3}{*}{ Grey cast iron } & \multirow{3}{*}{5.6} & 2 & 162.03 \\
\cline { 3 - 4 } & 2.5 & 158.62 \\
\cline { 2 - 4 } & \multirow{3}{*}{6.6} & 3 & 154.6 \\
\cline { 2 - 4 } & & 2.5 & 156.27 \\
\cline { 3 - 4 } & & 3 & 152.75 \\
\cline { 3 - 4 } & & & 152.8 \\
\hline
\end{tabular}


- Structural analysis

3. For the gap between the successive fins: 5.6

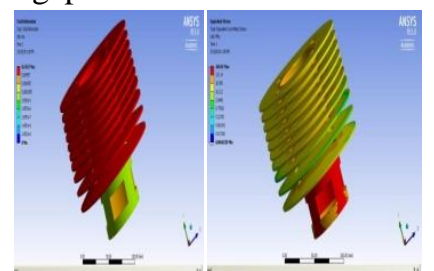

For fin thickness: $2 \mathrm{~mm}$

Fig 14

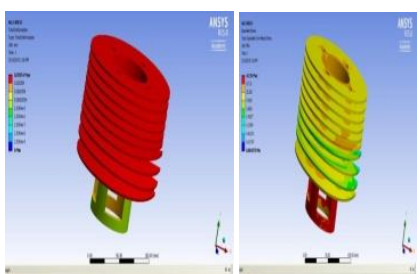

For fin thickness: $2.5 \mathrm{~mm}$

Fig 15

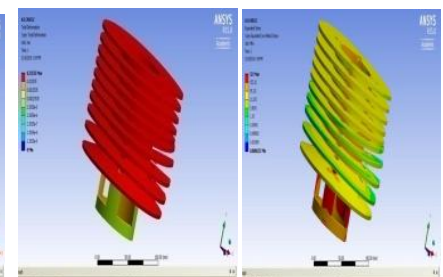

For fin thickness:3mm Fig 16

4. For the gap between the successive fins: $6.6 \mathrm{~mm}$

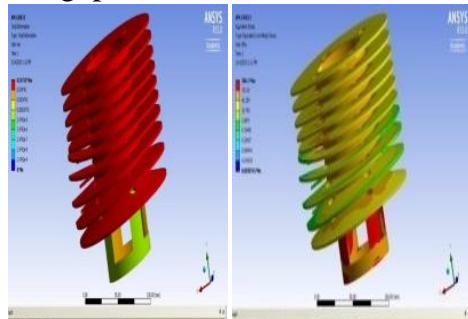

For fin thickness:2mm Fig 17

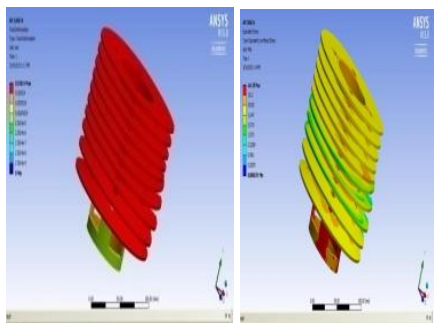

For fin thickness: $2.5 \mathrm{~mm}$

Fig .18

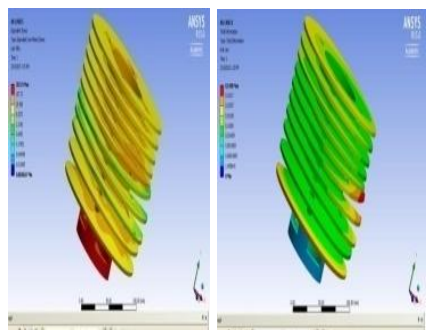

For fin thickness: $3 \mathrm{~mm}$ Fig.19

Table 5: static structural:

\begin{tabular}{|c|c|c|c|c|}
\hline Material & \multirow{2}{\text{Gapbetween}}{$\begin{array}{l}\text { Gap } \\
\text { fins(mm) }\end{array}$} & $\begin{array}{l}\text { Fin } \\
\text { thickness(mm) }\end{array}$ & $\begin{array}{l}\text { Totaldeformati } \\
\text { on(mm) }\end{array}$ & $\begin{array}{l}\text { Equalent } \\
\text { stress(Mpa) }\end{array}$ \\
\hline \multirow{2nnyy}{*}{$\begin{array}{c}\text { Grey cast } \\
\text { iron }\end{array}$} & \multirow{2}{*}{5.6} & 2 & 0.24557 & 469.92 \\
\cline { 1 - 4 } & & 2.5 & 0.28354 & 412.94 \\
\cline { 3 - 5 } & & 3 & 0.23535 & 521 \\
\cline { 3 - 5 } & \multirow{2}{*}{6.6} & 2 & 0.24742 & 566.1 \\
\cline { 3 - 5 } & & 2.5 & 0.25024 & 444.78 \\
\cline { 3 - 5 } & & 3 & 0.2490 & 387.19 \\
\hline
\end{tabular}

4.6. Material: Magnesium alloy:Thermal analysis of cylinder

1) For the gap between the successive fins: $5.6 \mathrm{~mm}$

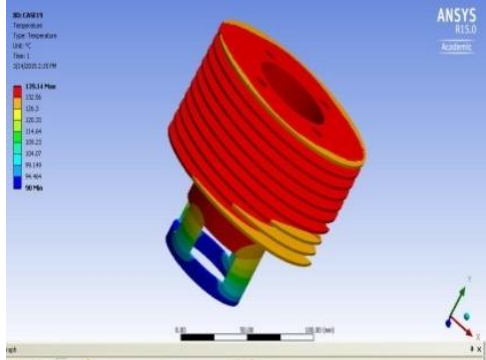

For Fin thickness: $2 \mathrm{~mm}$

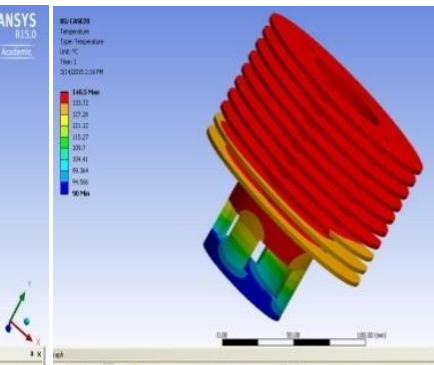

For fin thickness: $2.5 \mathrm{~mm}$

Fig 20

2) For the gap between the successive fins: $6.6 \mathrm{~mm}$

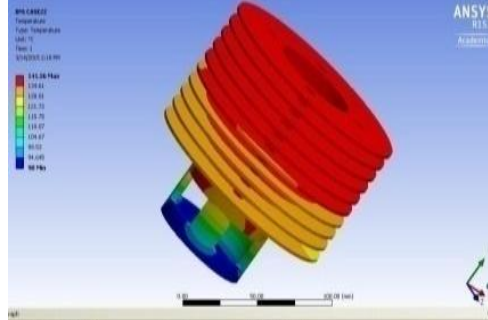

For Fin thickness: $2 \mathrm{~mm}$

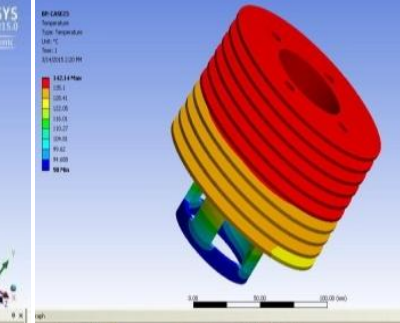

For fin thickness: $2.5 \mathrm{~mm}$

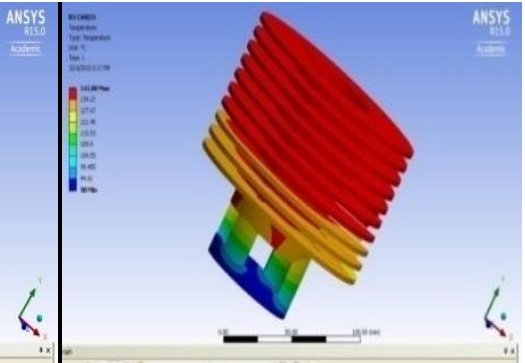

For fin thickness: $3 \mathrm{~mm}$

$$
\text { Fig } 21
$$


Table 6: Steady state thermal

\begin{tabular}{|c|c|c|c|}
\hline Material & $\begin{array}{l}\text { Gap between } \\
\text { fins(mm) }\end{array}$ & Fin thickness $(\mathbf{m m})$ & Temperature $\left({ }^{\circ} \mathbf{C}\right)$ \\
\hline \multirow{3}{*}{$\begin{array}{c}\text { megnesium } \\
\text { alloy }\end{array}$} & \multirow{2}{*}{5.6} & 2 & 139.4 \\
\cline { 2 - 3 } & & 2.5 & 140.5 \\
\cline { 2 - 4 } & \multirow{2}{*}{6.6} & 2 & 141.08 \\
\cline { 2 - 4 } & & 2.5 & 141.56 \\
\cline { 2 - 4 } & & 3 & 142.14 \\
\cline { 2 - 4 } & & & 142.56 \\
\hline
\end{tabular}

\section{- Structural analysis:}

1) For the gap between the successive fins: $5.6 \mathrm{~mm}$

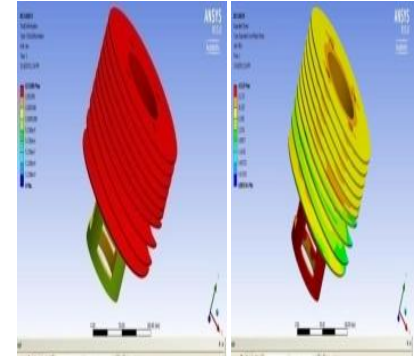

For fin thickness: $2 \mathrm{~mm}$

Fig 22

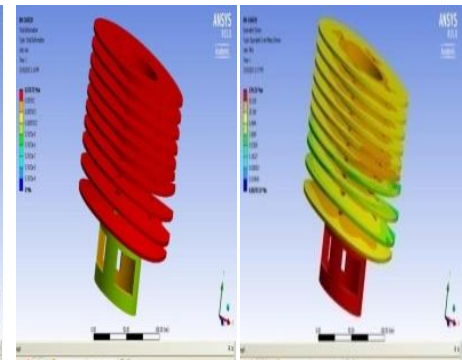

For fin thickness: $2.5 \mathrm{~mm}$ Fig 23

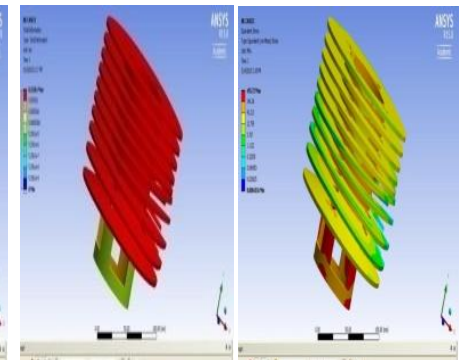

For fin thickness: $3 \mathrm{~mm}$

Fig 24

2) For the gap between the successive fins: $6.6 \mathrm{~mm}$

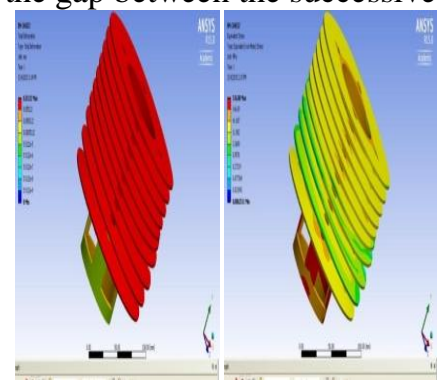

For fin thickness: $2 \mathrm{~mm}$

Fig 25

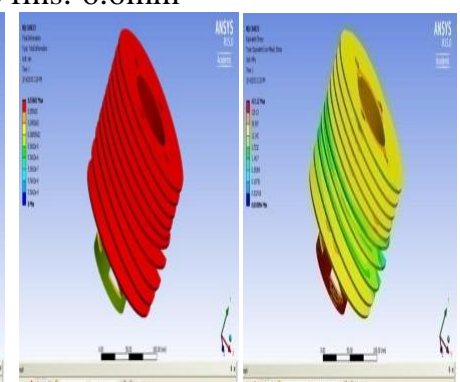

For fin thickness: $2.5 \mathrm{~mm}$

Fig 26

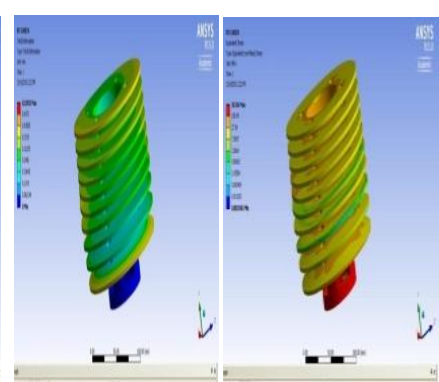

For fin thickness:3mm

Fig 27

Table 7: static structural

\begin{tabular}{|c|c|c|c|c|}
\hline Material & $\begin{array}{l}\text { Gapbetweenfins } \\
(\mathrm{mm}\end{array}$ & $\begin{array}{l}\text { Fin } \\
\text { thickness(mm) }\end{array}$ & $\begin{array}{l}\text { Totaldeformation( } \\
\text { mm) }\end{array}$ & $\begin{array}{l}\text { Equalentstress( } \\
\text { Mpa }\end{array}$ \\
\hline \multirow{6}{*}{$\begin{array}{l}\text { Megnisium } \\
\text { alloy }\end{array}$} & \multirow{3}{*}{5.6} & 2 & 0.53388 & 415.35 \\
\hline & & 2.5 & 0.57672 & 339.35 \\
\hline & & 3 & 0.52561 & 495.72 \\
\hline & \multirow{3}{*}{6.6} & 2 & 0.55122 & 516.08 \\
\hline & & 2.5 & 0.55602 & 421.12 \\
\hline & & 3 & 0.55935 & 367.04 \\
\hline
\end{tabular}

4.7 Material: Aluminium alloy:Thermal analysis of cylinder

1) For the gap between the successive fins: $5.6 \mathrm{~mm}$

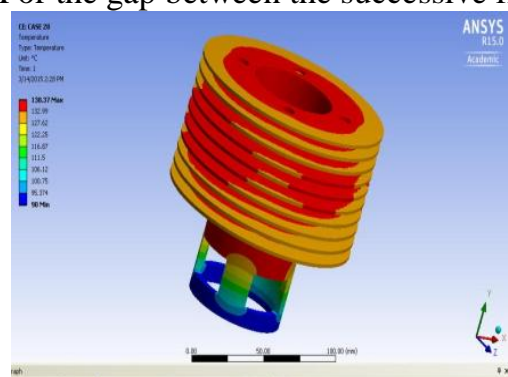

For Fin thickness: $2 \mathrm{~mm}$

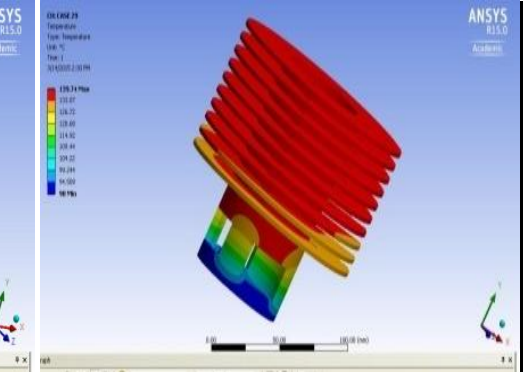

For fin thickness: $2.5 \mathrm{~mm}$

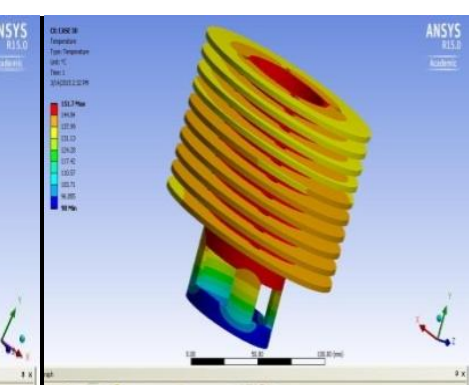

For fin thickness: $3 \mathrm{~mm}$

Fig 28 
2) For the gap between the successive fins: $6.6 \mathrm{~mm}$

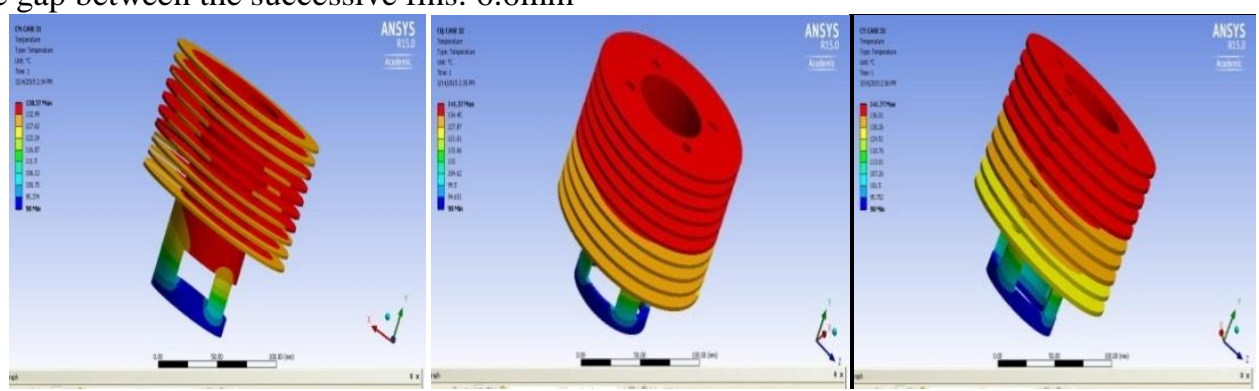

For Fin thickness : 2mm For fin thickness: $2.5 \mathrm{~mm}$ For fin thickness: $3 \mathrm{~mm}$

Fig 29

Table 8: Steady state thermal

\begin{tabular}{|c|c|c|c|}
\hline Material & $\begin{array}{l}\text { Gap between } \\
\text { fins(mm) }\end{array}$ & $\begin{array}{l}\text { Fin thickness } \\
(\mathbf{m m})\end{array}$ & Temperature $\left({ }^{\circ} \mathbf{C}\right)$ \\
\hline \multirow{2}{*}{$\begin{array}{c}\text { Alluminium } \\
\text { Alloy }\end{array}$} & \multirow{2}{*}{5.6} & 2 & 138.37 \\
\cline { 2 - 3 } & & 2.5 & 139.74 \\
\cline { 2 - 3 } & \multirow{2}{*}{6.6} & 2 & 151.7 \\
\cline { 2 - 4 } & & 2.5 & 138.37 \\
\cline { 2 - 4 } & & 3 & 141.37 \\
\cline { 2 - 4 } & & & 141.77 \\
\hline
\end{tabular}

- Structural analysis

3) For the gap between the successive fins: $5.6 \mathrm{~mm}$

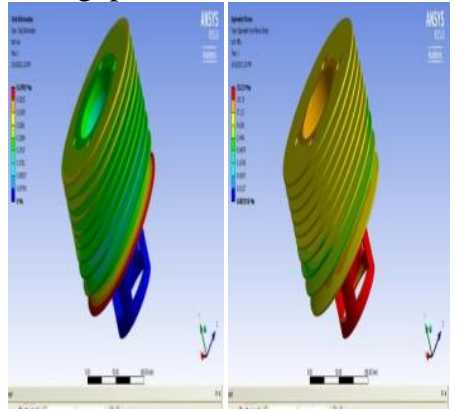

For fin thickness:2

Fig 30

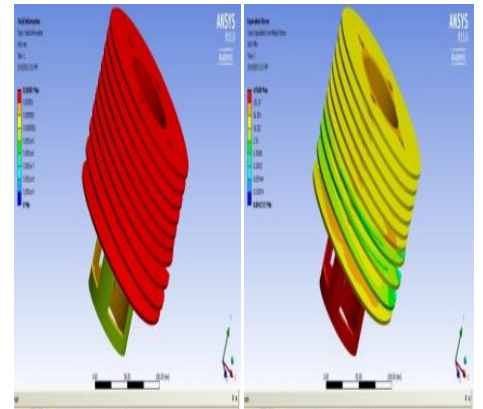

For fin thickness: $2.5 \mathrm{~mm}$ Fig.31

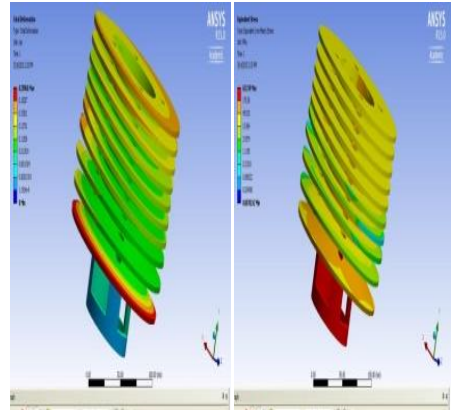

For fin thickness:3mm Fig 32

4) For the gap between the successive fins: $6.6 \mathrm{~mm}$

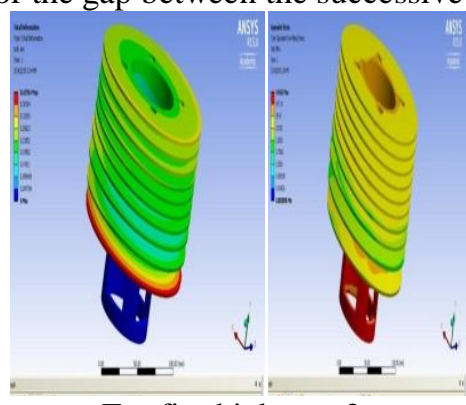

For fin thickness: $2 \mathrm{~mm}$

Fig 33

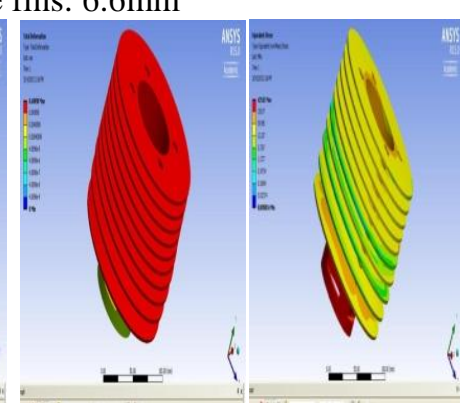

For fin thickness: $2.5 \mathrm{~mm}$

Fig 34

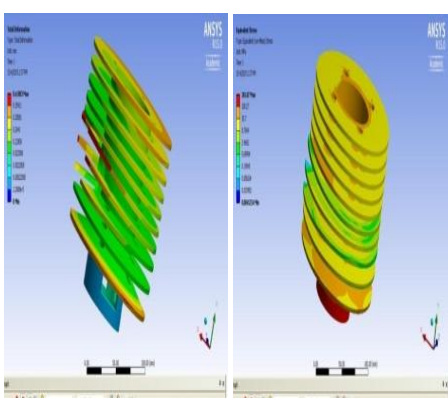

For fin thickness:3mm Fig 35

Table 9: static structural

\begin{tabular}{|c|c|c|c|c|}
\hline Material & Gapbetweenfins(mm) & Finthickness(mm) & Totaldeformation(mm) & Equalentstress(Mpa) \\
\hline \multirow{3}{*}{$\begin{array}{c}\text { Alluminium } \\
\text { alloy }\end{array}$} & \multirow{3}{*}{5.6} & 2 & 0.42992 & 552.33 \\
\cline { 2 - 4 } & & 2.5 & 0.50501 & 479.88 \\
\cline { 2 - 4 } & \multirow{3}{*}{6.6} & 3 & 0.20966 & 622.59 \\
\cline { 2 - 4 } & & 2 & 0.42934 & 549.65 \\
\cline { 2 - 4 } & & 2.5 & 0.40898 & 429.01 \\
\cline { 2 - 4 } & & 3 & 0.41003 & 381.87 \\
\hline
\end{tabular}




\section{Results}

The geometry of the original model is imported into ANSYS workbench 15.0 environment and boundary conditions were applied. Analysis is carried out for different geometry of fins (circular) with various thicknesses and different materials. The results are shown below

Table 10: Analysis Results

\begin{tabular}{|c|c|c|c|c|c|}
\hline Material & $\begin{array}{l}\text { Gap between fins } \\
(\mathrm{mm})\end{array}$ & $\begin{array}{l}\text { Fin } \\
\text { thickness } \\
(\mathbf{m m})\end{array}$ & $\begin{array}{l}\text { Temperature } \\
\left({ }^{\circ} \mathrm{C}\right)\end{array}$ & $\begin{array}{l}\text { Total } \\
\text { deformation } \\
(\mathrm{mm})\end{array}$ & $\begin{array}{l}\text { Equalent } \\
\text { stress(Mpa } \\
\text { ) }\end{array}$ \\
\hline \multirow{6}{*}{$\begin{array}{l}\text { Mild } \\
\text { Steel }\end{array}$} & \multirow{3}{*}{5.6} & 2 & 162.03 & 0.22957 & 917.57 \\
\hline & & 2.5 & 158.62 & 0.28668 & 827.62 \\
\hline & & 3 & 154.6 & 0.20869 & 660.87 \\
\hline & \multirow{3}{*}{6.6} & 2 & 156.27 & 0.22413 & 682.71 \\
\hline & & 2.5 & 152.75 & 0.20446 & 617.75 \\
\hline & & 3 & 152.8 & 0.19769 & 584.65 \\
\hline \multirow{6}{*}{$\begin{array}{c}\text { Grey } \\
\text { Cast iron }\end{array}$} & \multirow{3}{*}{5.6} & 2 & 162.03 & 0.24557 & 469.92 \\
\hline & & 2.5 & 158.62 & 0.28354 & 412.94 \\
\hline & & 3 & 154.6 & 0.23535 & 521 \\
\hline & \multirow[t]{3}{*}{6.6} & 2 & 156.27 & 0.24742 & 566.1 \\
\hline & & 2.5 & 152.75 & 0.25024 & 444.78 \\
\hline & & 3 & 152.8 & 0.2490 & 387.19 \\
\hline \multirow{6}{*}{$\begin{array}{c}\text { Magnesiu } \\
\text { m } \\
\text { Alloy }\end{array}$} & \multirow{3}{*}{5.6} & 2 & 139.4 & 0.53388 & 415.35 \\
\hline & & 2.5 & 140.5 & 0.57672 & 339.35 \\
\hline & & 3 & 141.08 & 0.52561 & 495.72 \\
\hline & \multirow[t]{3}{*}{6.6} & 2 & 141.56 & 0.55122 & 516.08 \\
\hline & & 2.5 & 142.14 & 0.55602 & 421.12 \\
\hline & & 3 & 142.56 & 0.55935 & 367.04 \\
\hline
\end{tabular}

\begin{tabular}{|c|c|c|c|c|c|}
\hline \multirow{3}{*}{$\begin{array}{c}\text { Alluminium } \\
\text { alloy }\end{array}$} & \multirow{3}{*}{5.6} & 2 & 138.37 & 0.42992 & 552.33 \\
\cline { 3 - 5 } & & 2.5 & 139.74 & 0.50501 & 479.88 \\
\cline { 3 - 5 } & 3 & 151.7 & 0.20966 & 622.59 \\
\cline { 2 - 5 } & \multirow{3}{*}{6.6} & 2 & 138.37 & 0.42934 & 549.65 \\
\cline { 3 - 5 } & 2.5 & 141.37 & 0.40898 & 429.01 \\
\cline { 3 - 5 } & 3 & 141.77 & 0.41003 & 381.87 \\
\hline
\end{tabular}

Table 11: Theoretical Results

\begin{tabular}{|c|c|c|c|c|}
\hline Material & $\begin{array}{l}\text { Finthickness(m } \\
\text { m) }\end{array}$ & $\begin{array}{l}\text { Heat lost } \\
\text { (w) }\end{array}$ & Effectiveness (E) & $\begin{array}{l}\text { Efficiency } \\
(\eta)\end{array}$ \\
\hline \multirow{3}{*}{$\begin{array}{l}\text { Mild } \\
\text { Steel }\end{array}$} & 2 & 83.72 & 16.05 & 82 \\
\hline & 2.5 & 86.52 & 16.05 & 84 \\
\hline & 3 & 89.35 & 16.05 & 86 \\
\hline \multirow{3}{*}{$\begin{array}{c}\text { Grey } \\
\text { Cast iron }\end{array}$} & 2 & 83.72 & 14.88 & 82 \\
\hline & 2.5 & 86.52 & 14.88 & 84 \\
\hline & 3 & 89.35 & 14.88 & 86 \\
\hline \multirow{3}{*}{$\begin{array}{l}\text { Magnesium } \\
\text { Alloy }\end{array}$} & 2 & 83.72 & 25.7 & 82 \\
\hline & 2.5 & 86.52 & 25.7 & 84 \\
\hline & 3 & 89.35 & 25.7 & 86 \\
\hline \multirow{3}{*}{$\begin{array}{l}\text { Alluminium } \\
\text { alloy }\end{array}$} & 2 & 83.72 & 26.51 & 82 \\
\hline & 2.5 & 86.52 & 26.51 & 84 \\
\hline & 3 & 89.35 & 26.51 & 86 \\
\hline
\end{tabular}

\section{Conclusion}

In this paper we have designed a cylinder fin body used in a 100cc Bajaj Motorcycle and modeled in ANSYS workbench 15.0 modeling software. Present used material for fin body is Aluminum alloy 204. We are replacing with Mild steel, grey cast iron, magnesium alloy, aluminum alloy. The shape of the fin is circular with rectangular cross section shaped. The default thickness of fin is $3 \mathrm{~mm}$; we are reducing it to $2,2.5 \mathrm{~mm}$. By reducing the thickness and also by changing the gap between the successive fins. thereby increasing the efficiency. The weight of the fin body is reduced when Magnesium alloy is used. We have done thermal analysis on the fin body by varying materials, geometry and thickness. By observing the analysis results, using circular fin, material Aluminum alloy and thickness of $2.5 \mathrm{~mm}$ is better since heat transfer rate is more. But by using circular fins the weight of the fin body increases.. So we can conclude that using material Aluminum alloy is better, reducing thickness to $2.5 \mathrm{~mm}$ is better and using fin shape circular by analysis By using circular fins the weight of the fin body reduces compared to existing rectangular engine cylinder fin. We have also done 
theoretical calculations to determine the heat lost, effectiveness and efficiency of the fins. By observing the results, using circular fins the heat lost is more, efficiency and effectiveness is also more

\section{References}

[1]. Rosli abu bakar, devarajan ramasamy chiew chen wee "Heat transfer in the cylinder of a new two stroke cross scavenged engine",Malaysia.

[2]. H.K.D.H Bhadeshia " Thermal analysis techniques" University of Cambridge,material science and metallurgy.

[3]. V.Esfahanian,A.Javaheri and M.Ghaffarpour "Thermal analysis of an S.I. engine. Using different combustion boundary condition treatment". United States (2005)

[4]. Qiang Du and Desheng Wang, "Tetrahedral mesh generation and optimization based on centroidal voronoi tessellations" PA 16802,U.S.A.

[5]. Mario Hirz; Tanja Goeber; Michal Ludwig; Michael Lang; Roland Kirchberger; Wolfgang Hirschberg. "Design and Development of a 50 Cc Scooter Frame Supported By Testing and Simulation." SAE International, Dated- October 2005.

[6]. M.Ayaz Afsar, "Experimental Investigation of direct air injection scavenged two strokk engine". Proceedings of 2009 International Symposium on Computing communication and control, Singapore. ISBN 978-1-84626.

[7]. Ibrahim, Semin ,Rosli Abu Bakar."In-cylinder mass flow rate and gas species concentration simulation of S.I.engine'ISSN.1816-949X,2007,Malaysia.

[8]. V.Ganesan, Internal combustion engines second edition Tata McGraw Hill publication, New Delhi, 2005.pp.1-6.

[9]. M.L.Mathur and R.P.Sharma.Internal combustion engine Dhanpat Rai New Delhi 2007 pp.164 to 183

[10]. R.K. Rajput, Heat and Mass Transfer, third edition S.Chand publication, New Delhi, 2006.pp.

[11]. 11-16 11] R.K. Rajput, Thermal engineering, sixth edition Laxmi publication, New Delhi 2006, pp945-957. 